\title{
AtEAF1 is a potential platform protein for Arabidopsis NuA4 acetyltransferase complex
}

\author{
Tomasz Bieluszewski', Lukasz Galganski', Weronika Sura', Anna Bieluszewska², Mateusz Abram', \\ Agnieszka Ludwikow ${ }^{1}$, Piotr Andrzej Ziolkowski ${ }^{1,3^{*}}$ and Jan Sadowski ${ }^{1 *}$
}

\begin{abstract}
Background: Histone acetyltransferase complex NuA4 and histone variant exchanging complex SWR1 are two chromatin modifying complexes which act cooperatively in yeast and share some intriguing structural similarities. Protein subunits of NuA4 and SWR1-C are highly conserved across eukaryotes, but form different multiprotein arrangements. For example, the human TIP60-p400 complex consists of homologues of both yeast NuA4 and SWR1-C subunits, combining subunits necessary for histone acetylation and histone variant exchange. It is currently not known what protein complexes are formed by the plant homologues of NuA4 and SWR1-C subunits.
\end{abstract}

Results: We report on the identification and molecular characterization of AtEAF1, a new subunit of Arabidopsis NuA4 complex which shows many similarities to the platform protein of the yeast NuA4 complex. AtEAF1 copurifies with Arabidopsis homologues of NuA4 and SWR1-C subunits ARP4 and SWC4 and interacts physically with AtYAF9A and AtYAF9B, homologues of the YAF9 subunit. Plants carrying a T-DNA insertion in one of the genes encoding AtEAF1 showed decreased FLC expression and early flowering, similarly to Atyaf9 mutants. Chromatin immunoprecipitation analyses of the single mutant Ateafib-2 and artificial miRNA knock-down Ateaf1 lines showed decreased levels of H4K5 acetylation in the promoter regions of major flowering regulator genes, further supporting the role of AtEAF1 as a subunit of the plant NuA4 complex.

Conclusions: Growing evidence suggests that the molecular functions of the NuA4 and SWR1 complexes are conserved in plants and contribute significantly to plant development and physiology. Our work provides evidence for the existence of a yeast-like EAF1 platform protein in A. thaliana, filling an important gap in the knowledge about the subunit organization of the plant NuA4 complex.

Keywords: NuA4, EAF1, YAF9, Arabidopsis thaliana, histone acetylation, PIE1

\section{Background}

Eukaryotic chromatin has evolved for seemingly contradictory functions. It ensures compaction and protection of genetic material, but also controls diverse processes including transcription, replication and DNA repair that require a relatively open and dynamic chromatin structure. This mixture of robustness and flexibility is achieved by a number of specialized enzymes that remodel chromatin or modify nucleosomes by covalent histone modifications. Different chromatin modifications can have a combinatorial effect on chromatin properties and

\footnotetext{
*Correspondence: paz22@cam.ac.uk; jsad@amu.edu.pl

'Department of Biotechnology, Institute of Molecular Biology and Biotechnology, Adam Mickiewicz University, Umultowska 89, 61-614 Poznań, Poland

Full list of author information is available at the end of the article
}

influence each other by guiding, stimulating or inhibiting chromatin modifying enzymes.

One of the best studied examples of an interplay between different types of chromatin modifications is the strong functional relationship between histone acetylation and chromatin remodeling. Protein domains specialized in specific recognition of acetylated histone tails are often found in chromatin remodeling complexes. Acetylation of nucleosomal histones has a strong influence on the action of chromatin remodeling complexes such as RSC [1], SWI/SNF [2], INO80 [3] or SWR1-C [4].

In the case of SWR1-C, this link seems to go much further. In yeast, the histone variant exchange reaction, catalyzed by SWR1-C is stimulated by the NuA4 complex through acetylation of nucleosomal histones [4]. These two protein complexes not only cooperate, but also share 
interesting structural similarities. Each of the two complexes is formed by more than ten different protein subunits organized into modules [5]. One of the modules, composed of proteins ARP4, SWC4, YAF9 and monomeric Actin, is common to both complexes [6]. Furthermore, a crucial role in the integrity of NuA4 and SWR1-C is played by proteins EAF1 and SWR1, respectively, which organize different modules into a functional complex. Although EAF1 lacks the ATPase domain, central to the chromatin remodeling activity of SWR1-C, it shares with SWR1 the HSA domain which is necessary for binding of the common module ARP4-SWC4-YAF9-Actin [5,7].

In human, homologues of yeast NuA4 and SWR1-C subunits form a hybrid complex called TIP60-p400. How far the functions of the fused complexes are conserved is yet to be determined. Whereas the ATPase activity of p400 enables H2A.Z deposition through histone exchange [8], the HAT activity of the TIP60 subunit seems to be blocked by the association with p400 [9]. Nevertheless, subunit conservation between SWR1, NuA4 and TIP60-p400 implies a strong evolutionary link. One attractive explanation of the relationship between SWR1-C, NuA4 and TIP60-p400 points to the fact that the integrity of the three complexes depends on their platform subunits SWR1, EAF1 and p400, respectively. The complex is formed when the remaining subunits bind, directly or as a part of a protein module, to several conserved features of the platform protein. Strikingly, p400 combines the features of SWR1 and EAF1. A synthetic p400-like construct, obtained by insertion of the ATPase domain of SWR1 between the HSA (helicase/SANT-associated) and SANT (Swi3, Ada2, $\mathrm{N}$-Cor, and TFIIIB) domains of EAF1, reconstituted a TIP60-p400-like complex when expressed in yeast [5]. The authors of the study concluded that a similar rearrangement could have given rise to the p400-like architecture in higher eukaryotes [5].

Outside Metazoa, the best characterized example of a domain architecture similar to that of p400 is PIE1, a protein necessary for incorporation of H2A.Z into nucleosomes in Arabidopsis thaliana [10,11]. HSA, ATPase and SANT domains are all present in PIE1 and show a high degree of sequence similarity to the corresponding domains of $\mathrm{p} 400$. Although most of the subunits of NuA4 and SWR1-C have clear homologues in Arabidopsis, no study has addressed the question of whether PIE1 can organize these proteins into a hybrid complex similar to TIP60-p400. Importantly, a plant homologue of EAF1 has not been identified so far, which calls into question the existence of an independent NuA4 complex in plants.

Up to now, studies in plants have embraced homologues of only three subunits of the yeast $\mathrm{NuA} 4$ complex, ESA1, YAF9 and EAF3. ESA1, the only essential histone acetyltransferase in S. cerevisiae and the catalytic subunit of the NuA4 complex [12], has two homologues in A. thaliana, HAM1 (Histone Acetyltransferase of the MYST family 1) and HAM2. Both proteins specifically acetylate lysine 5 of the histone $\mathrm{H} 4$ [13] and are functionally redundant, as the single mutants display no developmental phenotype, while a double mutant is nonviable [14]. Reduction of HAM1 and HAM2 transcript levels results in decreased expression of the negative flowering regulator FLOWERING LOCUS C (FLC) and premature transition to flowering. This change is accompanied by a decrease in $\mathrm{H} 4$ acetylation in the chromatin of FLC [15]. Similar effects were observed in plants deficient in AtYAF9A, one of the two Arabidopsis homologues of yeast YAF9, a subunit shared by NuA4 and SWR1-C [16]. Interestingly, a recent study shows that simultaneous loss of function of two A. thaliana homologues of EAF3 results in late flowering, also mediated by reduced histone acetylation which, in this case, disrupts the expression of flowering inducer FLOWERING LOCUS T (FT) [17].

The aim of this study was to determine whether Arabidopsis homologues of NuA4 subunits are organized into a big protein complex similar to yeast $\mathrm{NuA} 4$ or human TIP60-p400. Our initial hypothesis was that PIE1 serves as a platform protein for a plant analog of the human TIP60-p400 complex. Through analysis of proteins that bind to Arabidopsis homologues of ARP4 and SWC4, common subunits of yeast NuA4 and SWR1-C, we confirmed their interaction with PIE1 and other Arabidopsis homologues of NuA4 and SWR1-C subunits. In addition, we revealed their association with an uncharacterized EAF1-like protein, not previously considered a subunit of plant NuA4 or SWR1-C. Subsequently, we focused on a possible role of AtEAF1 as a platform of the plant NuA4 complex. We demonstrated that one of the two isoforms of AtEAF1 interacts with AtYAF9A and AtYAF9B through a conserved HSA domain. Phenotypical analyses of mutants indicated that AtEAF1 and AtYAF9 are necessary for proper timing of transition to flowering, which can be explained by their influence on the H4K5 acetylation in the chromatin of major flowering regulator genes and their transcriptional activity, supporting the role of AtEAF1 as a platform subunit in the plant NuA4 complex.

\section{Results}

An uncharacterized plant-specific domain-relative of the yeast EAF1 protein is physically associated with AtARP4 and AtSWC4

We used affinity purification followed by tandem mass spectrometry (AP-MS/MS) to test which Arabidopsis homologues of yeast NuA4 and SWR1-C are associated with the common protein module of the two complexes. For this purpose we chose Arabidopsis homologues of ARP4 and SWC4 as protein baits, because these subunits 
are encoded by single genes in A. thaliana. We overexpressed AtARP4 and AtSWC4 fused to Strep-tag, in an Arabidopsis cell suspension culture. Following purification of proteins bound to the baits, we identified them by mass spectrometry. Proteins detected in control purifications, with no bait or with Strep-GFP as bait, were eliminated as nonspecific hits (Additional file 1). Next, we looked for proteins showing sequence- or domain architecturesimilarity to subunits of NuA4 and SWR1-C. We also looked for homologues of INO80 complex subunits, because the yeast and human versions of this complex also contain ARP4 [18,19]. As expected, we found conserved subunits of all three complexes in association with AtARP4, while AtSWC4 copurified only with subunits of NuA4 and SWR1-C (Table 1). In agreement with a recently published study [20], we found multiple subunits of the SWI/SNF complex among proteins copurified with AtARP4, suggesting that besides NuA4, SWR1-C and INO80, AtARP4 interacts with SWI/SNF in plants. We identified no subunits characteristic of INO80 or SWI/SNF among the proteins copurified with AtSWC4, which additionally confirms the specificity of the detected interactions.

According to published data for other species, the presence of ARP4 and SWC4 subunits is restricted to protein complexes closely related to NuA4, SWR1-C and TIP60-p400. Therefore, we considered proteins copurified with both baits as potential subunits of a hypothetical plant TIP60-p400-like complex (Table 1). One uncharacterized protein in this category had a domain architecture similar to that of yeast EAF1 (Figure 1a). We therefore named it AtEAF1.

We aligned the amino acid sequences of AtEAF1 homologues identified in distantly related plant species with the sequences of $S$. cerevisiae EAF1 and its Schizosaccharomyces pombe homologue VID21, as well as with human p400 and several plant homologues of PIE1 (Figure 1a). Strikingly, all proteins contained highly conserved HSA and SANT domains (Figure 1b), despite little overall sequence similarity and different domain arrangements (Figure 1c).

AtEAFf 1 is encoded by two nearly identical genes that are both transcriptionally active in $A$. thaliana

AtEAF1 is encoded by two genes in all of the sequenced A. thaliana ecotypes, except Mt-0 (1001genomes.org). The two genes occupy adjacent loci At3g24870 and At3g24880 on the reverse strand of chromosome 3 and share $98.5 \%$ identity in their coding regions. As no fulllength cDNA clone was available for AtEAF1, we designed primers using existing annotations (Additional file 2), and cloned the full-length coding sequence (CDS) (Additional file 3). Our AtEAF1B CDS clone corresponds to the At3g24870.1 gene model (Additional file 4).
To determine whether both genes were transcriptionally active, we designed three pairs of primers complementary to both coding sequences. Each amplicon contained a restriction site specific for one gene (i.e. not found in the other). The results of restriction digestion of the RT-PCR products were consistent for all three amplicons and indicated that both transcripts are equally abundant in mature rosette leaves (Additional file 4).

\section{Plant homologues of the NuA4 complex subunit YAF9 interact with AtSWC4 and AtEAF1}

As shown above, we found evidence for physical association of AtARP4 and AtSWC4 with AtEAF1, a domain relative of yeast NuA4 subunit EAF1. We assumed that AtARP4 and AtSWC4 associated with AtEAF1 through interaction with the latter's HSA domain. Cooperative binding of ARP4 and SWC4 to the N-terminal region of SWR1, containing the HSA domain, requires YAF9 in yeast [7]. One of the two Arabidopsis homologues of YAF9 was shown recently to be required for histone $\mathrm{H} 4$ acetylation in the $F L C$ locus, in line with its role as a plant NuA4 subunit [16]. To see whether the HSA domain of AtEAF1 can recruit $A$. thaliana YAF9, we transiently coexpressed nEYFP-tagged AtYAF9A or AtYAF9B with the HSA-containing fragment of AtEAF1 fused to the Flag-tag in Arabidopsis mesophyll protoplasts. Coimmunoprecipitation showed that AtYAF9A and AtYAF9B do indeed interact with this fragment of AtEAF1 (Figure 2a, Additional file 5). We also tested HSA-containing fragments of PIE1 and AtINO80 fused to Flag-tag, but no interaction was observed.

\section{AtYAF9A and AtYAF9B interact with AtSWC4 in the nucleus}

Our AP-MS/MS results revealed a physical association of AtARP4 and AtSWC4 with AtYAF9A and AtYAF9B. To test whether AtARP4, AtSWC4, AtYAF9A and AtYAF9B associate closely to form a functional module, we screened these proteins for protein-protein interactions, using Bimolecular Fluorescence Complementation (BiFC) in Arabidopsis mesophyll protoplasts. Coexpression of AtYAF9A or AtYAF9B fused to the N- or C-terminal fragment of Enhanced Yellow Fluorescent Protein (EYFP) with AtSWC4 fused to the complementary EYFP fragment produced strong fluorescence localized in the nucleus (Figure 2b,c). No other combination of complementary fusions produced detectable EYFP fluorescence (Additional file 6).

Although pairwise sequence alignment between AtYAF9A and AtYAF9B amino acid sequences shows a high degree of similarity along their whole length, only a shorter splice variant of AtYAF9B has been studied previously $[16,21]$. We obtained CDS clones of both splice variants (Additional file 3, Additional file 4) and found that the shorter variant lacks the conserved $\mathrm{C}$-terminal region, 
Table $1 \mathrm{~A}$ thaliana homologues of yeast SWR1-C and NuA4 subunits copurify with AtARP4 and AtSWC4

\begin{tabular}{|c|c|c|c|c|c|c|c|c|c|c|c|}
\hline \multicolumn{2}{|c|}{ Purified proteins } & \multicolumn{2}{|l|}{ Homologues } & \multicolumn{2}{|l|}{ Baits } & \multicolumn{3}{|c|}{ Protein complexes } & \multicolumn{3}{|c|}{ Protein domains } \\
\hline Locus ID & Name & H. sapiens & S. cerevisiae & At ARP4 & At SWC4 & SWR1/NuA4 & INO80 & SWI/SNF-type & HSA & ATPase & SANT \\
\hline AT1G18450 & AtARP4 $^{3}$ & BAF53A & ARP4 & + & + & + & + & + & & & \\
\hline AT5G22330 & RVB1 & RUVBL1 & RVB1 & + & + & + & + & & & & \\
\hline AT5G67630 & RVB21 & RUVBL2 & RVB2 & + & + & + & + & & & & \\
\hline AT3G49830 & RVB22 & RUVBL2 & RVB2 & + & + & + & + & & & & \\
\hline АT3G24880 & AtEAF1A & - & EAF1 & + & + & + & & & + & & + \\
\hline АT3G24870 & AtEAF1B & - & EAF1 & + & + & + & & & + & & + \\
\hline AT4G14385 & EAF6 & MEAF6 & EAF6 & + & + & + & & & & & \\
\hline AT1G79020 & EPL1B & EPC1 & EPL1 & + & + & + & & & & & \\
\hline AT5G64610 & $\mathrm{HAM}^{1}$ & TIP60 & ESA1 & + & + & + & & & & & \\
\hline AT1G54390 & ING2 & ING3 & YNG2 & + & + & + & & & & & \\
\hline AT3G12810 & $\mathrm{PIE}^{1}$ & p400 & SWR1 & + & + & + & & & + & + & + \\
\hline AT2G47210 & AtSWC4 & DMAP1 & SWC4 & + & + & + & & & & & + \\
\hline AT5G45600 & AtYAF9A ${ }^{1}$ & GAS41 & YAF9 & + & + & + & & & & & \\
\hline АТЗG33520 & ARP6 $^{1}$ & ARP6 & ARP6 & & + & + & & & & & \\
\hline AT4G37280 & $M R G 1^{1}$ & MRG15 & EAF3 & & + & + & & & & & \\
\hline AT1G26470 & EAF7 & MRGBP & EAF7 & & + & + & & & & & \\
\hline AT1G16690 & EPL1A & EPC1 & EPL1 & & + & + & & & & & \\
\hline AT2G36740 & $\mathrm{SWC2}{ }^{1}$ & $Y L-1$ & SWC2 & & + & + & & & & & \\
\hline AT5G37055 & sWC6 ${ }^{1}$ & ZNHIT1 & sWC6 & & + & + & & & & & \\
\hline AT4G36080 & TRA1 & TRRAP1 & TRA1 & & + & + & & & & & \\
\hline AT2G18000 & AtYAF9B ${ }^{1}$ & GAS41 & YAF9 & & + & + & & & & & \\
\hline AT3G12380 & $\mathrm{ARP5}^{2}$ & ARP5 & ARP5 & + & & & + & & & & \\
\hline AT5G43500 & ARP9 & ARP8 & ARP8 & + & & & + & & & & \\
\hline AT3G57300 & $\mathrm{INO}^{2} \mathrm{C}^{2}$ & INO80 & INO80 & + & & & + & & + & + & \\
\hline AT5G13950 & NFRKB & NFRKB & - & + & & & + & & & & \\
\hline AT1G65650 & $\mathrm{UCH} 2$ & UCH37 & - & + & & & + & & & & \\
\hline AT5G16310 & $\mathrm{UCH} 2$ & UCH37 & - & + & & & + & & & & \\
\hline AT4G06634 & $Y Y 1$ & YY1 & - & + & & & + & & & & \\
\hline АT3G60830 & $\mathrm{ARP}^{3}$ & - & ARP7 & + & & & & + & & & \\
\hline АТЗG17590 & $\mathrm{BSH}^{3}$ & $|N| 1$ & SNF5 & + & & & & + & & & \\
\hline AT2G46020 & $\mathrm{BRM}^{3}$ & BRG1/HBRM & SNF2/STH1 & + & & & & + & + & + & \\
\hline AT2G28290 & $S Y D^{3}$ & BRG1/HBRM & SNF2/STH1 & + & & & & + & + & + & \\
\hline AT3G06010 & MINU1 & BRG1/HBRM & SNF2/STH1 & + & & & & + & + & + & \\
\hline AT5G19310 & MINU2 & BRG1/HBRM & SNF2/STH1 & + & & & & + & + & + & \\
\hline AT2G47620 & SWI3A ${ }^{3}$ & BAF155/BAF170 & SWI3 & + & & & & + & & & + \\
\hline AT2G33610 & SWI3B ${ }^{3}$ & BAF155/BAF170 & SWI3 & + & & & & + & & & + \\
\hline AT1G21700 & SWI $3 \mathrm{C}^{3}$ & BAF155/BAF170 & SWI3 & + & & & & + & & & + \\
\hline AT4G34430 & $S W I 3 D^{3}$ & BAF155/BAF170 & SWI3 & + & & & & + & & & + \\
\hline AT3G01890 & SWP73A & BAF60A & SNF12 & + & & & & + & & & \\
\hline AT5G14170 & SWP73B & BAF60A & SNF12 & + & & & & + & & & \\
\hline
\end{tabular}

Proteins copurified with AtARP4 or AtSWC4, fused to Strep-tag, were identified by MS/MS and manually annotated on the basis of sequence- and domain architecture-similarity to yeast and human proteins. The table is limited to proteins that were identified as subunits of chromatin remodeling or histone modifying complexes (multiple subunits were identified). Columns on the right show the distribution of selected protein domains.

${ }_{1,2,3}$ Proteins either directly or indirectly linked to Arabidopsis SWR1 and/or NuA4 (1), INO80 (2) or SWI/SNF-type (3) complexes, according to published experimental data. 


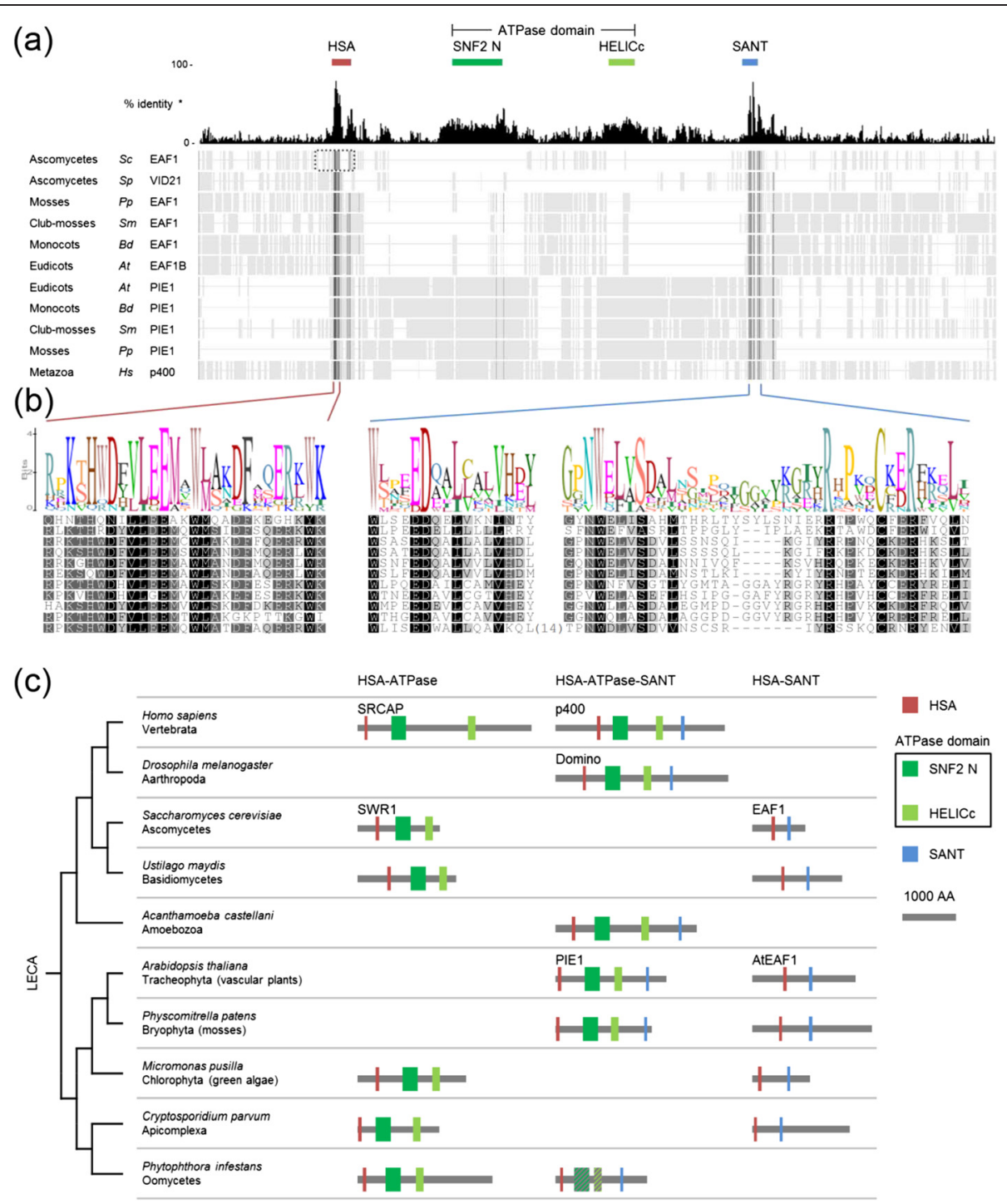

Figure 1 Arabidopsis thaliana AtEAF1 and PIE1 represent two distinct protein families that share highly similar HSA and SANT domains and do not coexist outside plants. (a) Protein alignment of domain relatives of Arabidopsis thaliana (At) AtEAF1 and PIE1, found in

Saccharomyces cerevisiae (Sc), Schizosaccharomyces pombe (Sp), Brachypodium distachyon (Bd), Selaginella moellendorffii (Sm), Physcomitrella patens $(P p)$ and Homo sapiens $(H s)$. A dotted rectangle depicts the region of yeast EAF1 that recruits ARP4 and actin, according to Szerlong et al. [28]. Columns containing over $75 \%$ gaps were removed from the alignment for clarity. Similarity shading of the alignments in (a) and (b) was done using the Blosum62 matrix. White indicates < $60 \%$ similarity, light grey 60 to $80 \%$, dark grey 80 to $100 \%$ and black $100 \%$. (b) A close-up of the conserved fragments of the HSA domain and the SANT domain. (c) Protein domains HSA, ATPase and SANT form three different domain architectures, which occur in various combinations across eukaryotes. All sequences and alignments used in this figure can be found in the Additional file 3. ${ }^{*}$ Mean pairwise identity over all pairs in the column. A sliding window of 5 was used in the histogram.

responsible for SWC4 binding in yeast [22]. In agreement with this observation, the shorter splice variant of AtYAF9B did not interact with AtSWC4 when tested by BiFC (Figure 2b). Therefore, we used the longer splice variant in all protein-protein interaction assays described herein.

AtEAF1B, AtYAF9A and AtYAF9B are necessary for normal FLC levels and timing of flowering

AtYAF9A-deficient plants express FLC at reduced levels [16]. Physical interaction between AtYAF9A and AtEAF1, and their similarity to functional counterparts in yeast, YAF9 and EAF1, respectively, suggested that AtEAF1 might also influence FLC transcription. To test this prediction we used plants with a T-DNA insertion near the $5^{\prime}$ end of the last exon of the AtEAF1B gene (Figure 3a, Additional file 4). We will refer to this line as Ateaf1b-2. Compared to wild-type seedlings, Ateaf1b-2 mutants expressed FLC at significantly reduced levels under both long day (LD) and short day (SD) conditions (Figure 3b). 


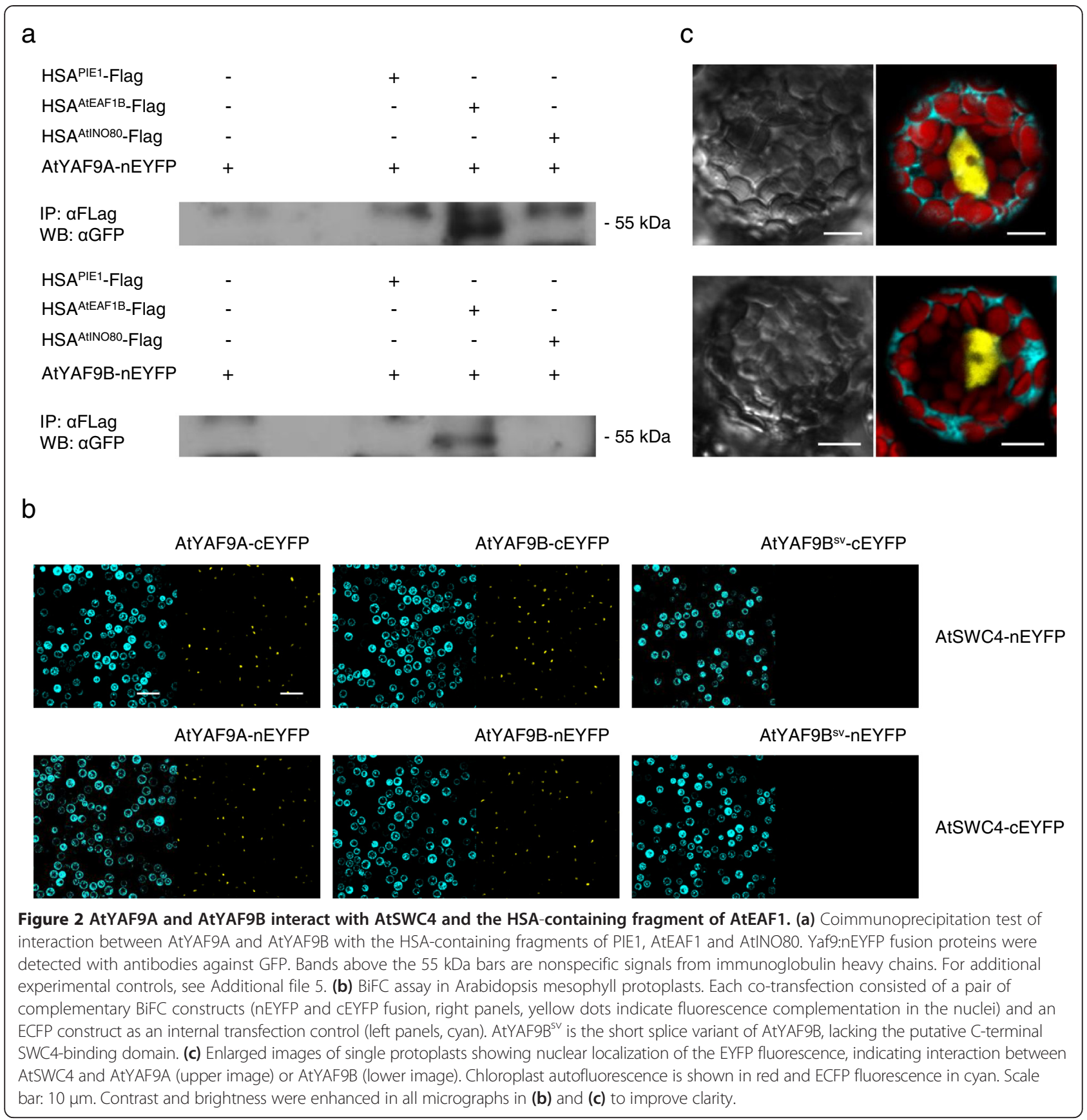

In our protein-protein interaction assays both AtYAF9A and AtYAF9B interacted with AtSWC4 and AtEAF1 (Figure 2). To investigate whether AtYAF9B also contributes to $F L C$ expression and whether there is a redundancy of AtYAF9A and AtYAF9B function, we compared the relative FLC expression levels of Atyaf9a-1, Atyaf $9 b-2$ and Atyaf $9 a-1$ Atyaf $9 b-2$ mutants with wild-type plants using the same experimental setup as described for Ateaf $1 b-2$ above. Interestingly, the FLC expression in the double mutant was not significantly reduced when compared to Atyaf9a-1 (LD p $=0.18$, $\mathrm{SD} \mathrm{p}=0.33$ ) (Figure 3b).

A decrease in FLC expression leads to earlier transition from vegetative to reproductive phase in the Atyaf $9 a-1$ mutant [16]. We tested the effect of reduced FLC expression on the timing of flowering in AtYAF9- and AtEAF1-deficient plants. We grew Atyaf9a-1, Atyaf $9 b-2$, Atyaf9a-1 Atyaf $9 b-2$ and Ateaf1b-2 mutants, as well as wild type plants, under LD and SD conditions. The Atyaf9a-1 Atyaf 9 b-2 double mutant flowered significantly 
(b)

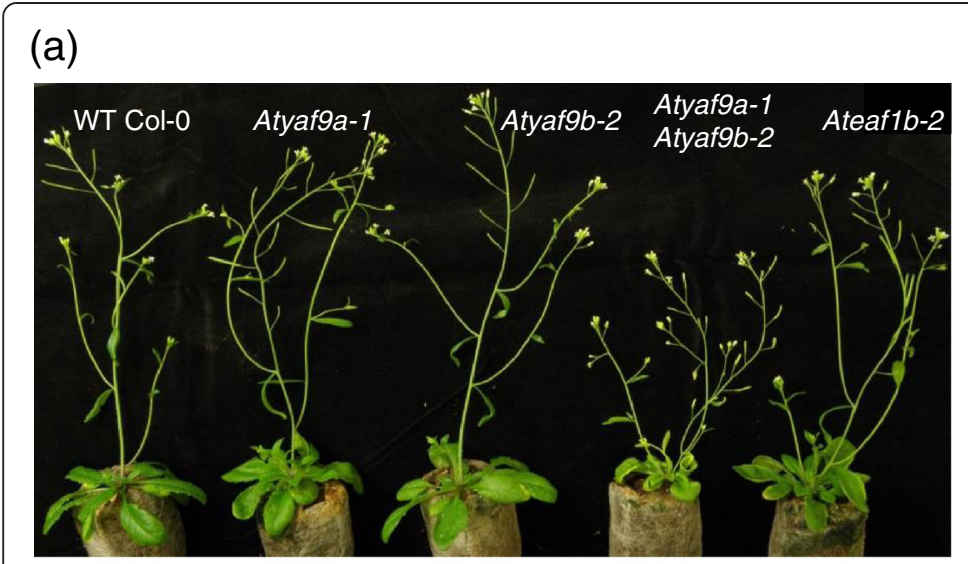

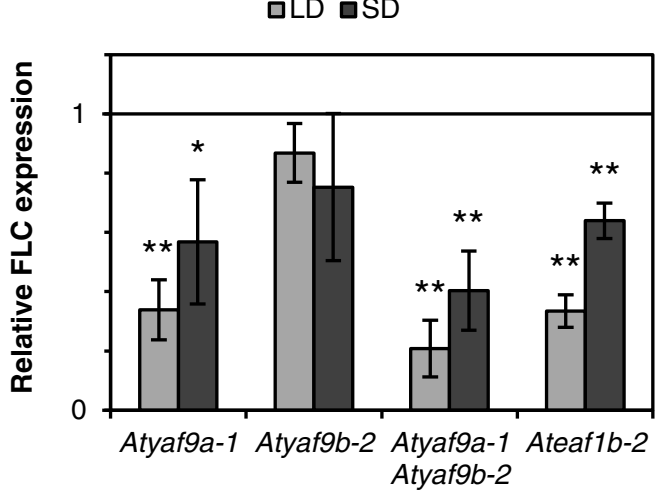

(c)
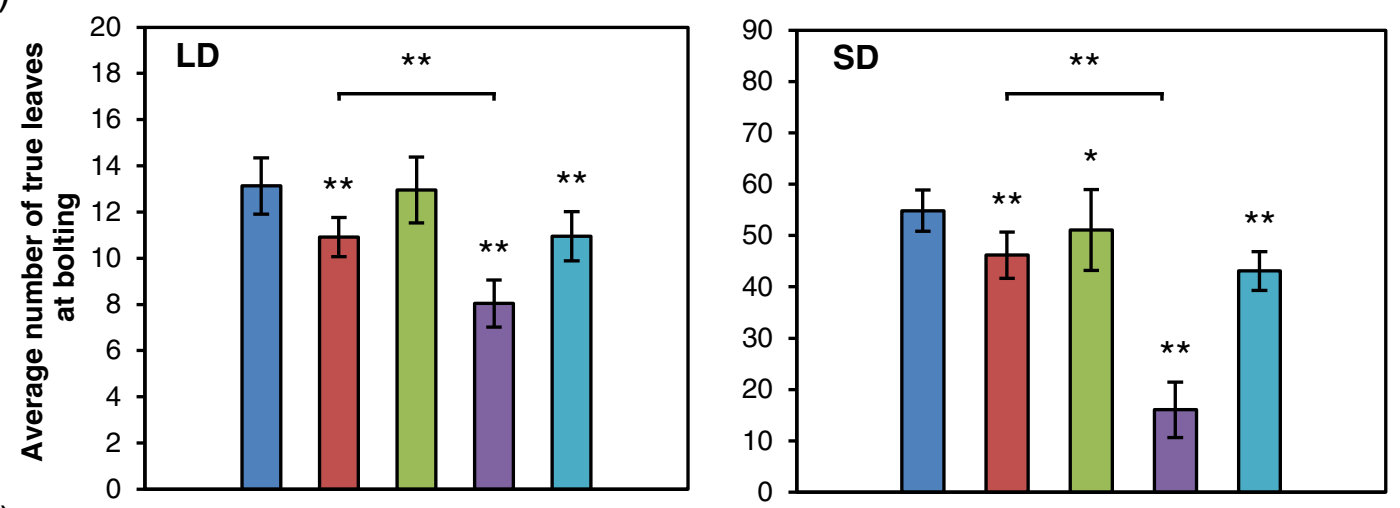

$\square W T$

口Atyaf9a-1

$\square$ Atyaf9b-2

$\square$ Atyaf9a-1 Atyaf9b-2

$\square$ Ateaf1b-2

(d)

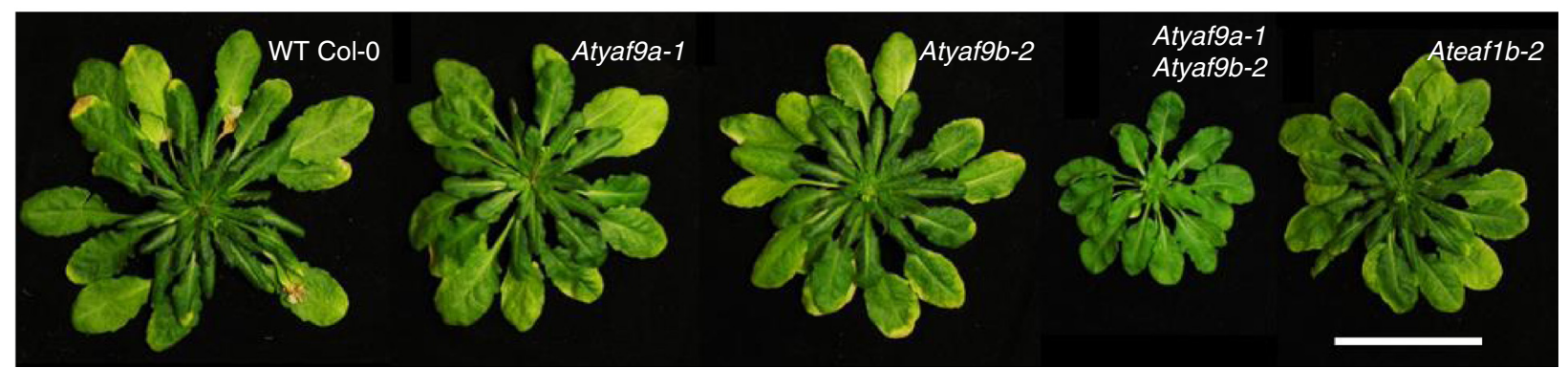

Figure 3 Mutations in AtEAF1 and AtYAF9 genes affect FLC expression and flowering time. (a) Comparison of plants grown under long day conditions (LD). (b) Relative expression levels of FLC transcript compared to WT control. Seedlings were collected in the middle of the light photoperiod. (c) Comparison of flowering time represented by an average number of true rosette leaves at the stage where the flower stem is $1 \mathrm{~cm}$ long. (d) Representative rosettes of plants grown under short day conditions (SD) at the stage when the leaves were counted. The bar length is $5 \mathrm{~cm}$. In all graphs, asterisks indicate statistical significance of the difference between each mutant and the WT control. A single asterisk indicates a p-value $<0.05$, double asterisk - $\mathrm{p}$-value $<0.01$ (t-test).

earlier than single mutants under both $\mathrm{LD}$ and SD conditions (Figure 3c,d). Importantly, both Ateaf1b-2 and Atyaf9a-1 mutants flowered at a similar growth stage, which was intermediate between that of the yaf9 double mutant and wild-type plants (WT), while the early flowering phenotype of the Atyaf $9 b-2$ mutant was least pronounced of all the mutant lines (Figure 3c).
AtEAF1 and AtYAF9 are necessary for normal levels of H4K5 acetylation

Published experimental data support involvement of Arabidopsis homologues of NuA4 subunits YAF9 and ESA1 (HAM1 and HAM2) in the acetylation of lysine 5 of histone H4 [13]. Therefore, if AtEAF1 is a functional subunit of the Arabidopsis NuA4 complex, partial loss of its function should lead to changes in H4K5 acetylation 
levels. As an initial test of the influence of AtEAF1 on H4K5 acetylation, we grew Ateaf1b-2 and Atyaf9a-1 Atyaf $9 b$ - 2 seedlings for 12 days on MS medium supplemented with $1 \%$ sucrose or $1 \%$ sucrose and $12.5 \mu \mathrm{M}$ Trichostatin A (TSA) (Figure 4). TSA is a specific inhibitor of histone deacetylases and has a strong negative effect on plant growth, coinciding with dramatic accumulation of acetylated histones [23,24]. We reasoned that impaired function of an important histone acetylatransferase such as $\mathrm{NuA} 4$ could prevent abnormal accumulation of acetylated histones and give mutant plants an advantage over WT plants under TSA challenge. As expected, average fresh weight of Atyaf9a-1 Atyaf $9 b-2$ and Ateaf1b-2 mutant plants grown on plates containing TSA was significantly larger than those of WT plants grown in the same conditions, whereas no significant differences where observed under control conditions (Figure 4b). In order to verify if the observed effect can be attributed to differences in global H4K5 acetylation levels, we tested the abundance of histone $\mathrm{H} 4$ acetylated on lysine 5 by Western Blot (Additional file 7). Only the double mutant Atyaf9a-1 Atyaf9b-2 displayed decreased levels of acetylated $\mathrm{H} 4 \mathrm{~K} 5$ relative to WT which indicates that the increased resistance of Ateafl $1 b$ - 2 plants to TSA cannot be due to a global loss of H4K5 acetylation. This observation could be explained if AtEAF1 had a specialized function in the Arabidopsis NuA4 complex. In fact, in yeast eaf1 mutant a strong decrease in histone $\mathrm{H} 4$ acetylation was observed in the promoter region of the $\mathrm{PHO} 5$ gene, but no decrease in bulk histone $\mathrm{H} 4$ acetylation was reported [5]. Therefore we decided to test if specific genomic targets of histone acetyltraferases are affected in plants with impaired function of AtEAF1. We focused on major regulators of flowering transition FLC, FT, CONSTANS (CO) and SUPPRESSOR OF OVEREXPRESSION OF CONSTANS 1 (SOC1) as histone acetylation in these genes was found to be deregulated by various $\mathrm{H} 4$ acetylation mutants in previous studies [15-17]. Our observations of flowering timing in Ateaf1b-2 mutant, presented above, further justified that choice.

In order to verify if the flowering phenotype of the Ateaf1b-2 mutant is related to the function of AtEAF1, we generated transgenic Arabidopsis plants in which transcript levels of $A t E A F 1 A$ and $A t E A F 1 B$ genes were reduced simultaneously through artificial micro RNA (amiRNA) (Figure 5a) [25]. Although independent transgenic lines expressing amiRNA construct displayed a moderate early flowering phenotype (Figure $5 \mathrm{~b}, \mathrm{c}$ ), it did not correlate with the decrease in AtEAF1 transcript (Figure 5), In the line 2.39 which showed the earliest flowering we detected only slightly decreased levels of AtEAF1 transcript while line 2.29, with stronger AtEAF1 silencing, showed less obvious early flowering phenotype.

Our next step was to characterize differences in histone H4K5 acetylation levels over FLC and FT genes between WT, Ateaflb-2, 2.29, 2.39 and Atyaf9a-1 Atyaf $9 b$-2 plants by chromatin immunoprecipitation (ChIP). We carried out the experiments on 10-day old seedlings grown under long day conditions, collected at the end of the day. For the amplification of the DNA fragments obtained from ChIP we used five pairs of PCR primers for each gene, corresponding to various functional elements of the gene (Figure 6a, b, Additional file 2). We observed a moderate but consistent decrease in H4K5 acetylation over both genes. Interestingly, we observed a stronger reduction in acetylation levels near the $5^{\prime}$ end of the genes, especially in the FLC locus. Following this observation, we tested the acetylation levels in the promoter regions of two other major flowering regulators, $\mathrm{CO}$ and SOC1. We observed a significant and consistent drop of $\mathrm{H} 4 \mathrm{~K} 5$ acetylation in the promoter of $\mathrm{CO}$ but little change in SOC1, except for the Atyaf9a-1 Atyaf9b-2 line, which showed significantly lower acetylation at both loci (Figure 7a).

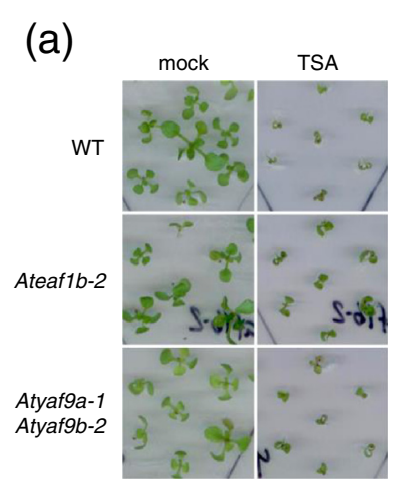

(b)

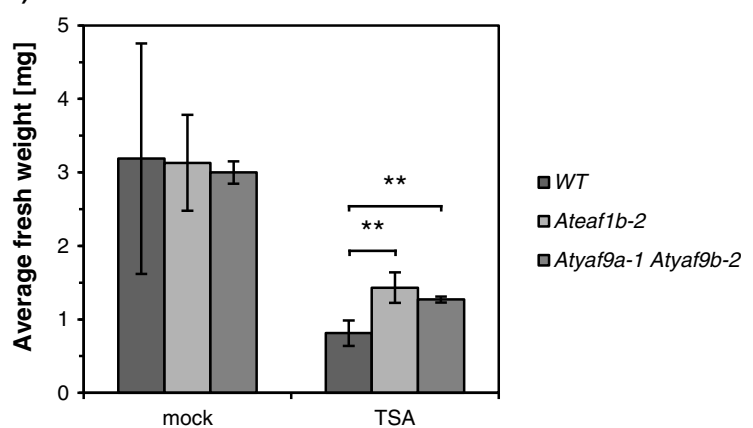

Figure 4 Ateaf1b-2 and Atyaf9a-1 Atyaf9b-2 mutants gain increased resistance to TSA. (a) 12 day-old seedlings grown in the presence of TSA or mock. All images are in the same scale. (b) Comparison of average fresh weight between plants treated with TSA or mock. Error bars represent standard deviation of 4 biological replicates. Double asterisks indicate a $\mathrm{p}$-value $<0.01$ (t-test). 
(a)

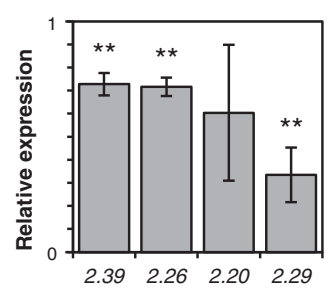

(b)

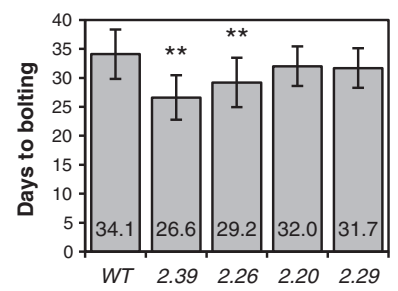

(c)

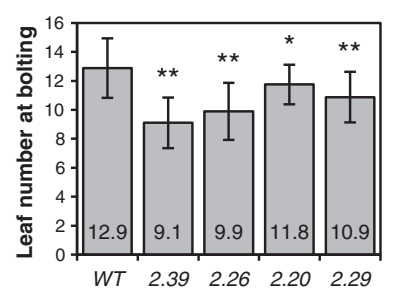

Figure 5 Plants with transcript levels of AtEAF1 decreased by artificial miRNA show deregulation of flowering time. (a) Expression levels of AtEAF1 in four independent amiRNA lines relative to WT Col-0. (b) Comparison of flowering time represented by an average number of days until the stage where the flower stem is $1 \mathrm{~cm}$ long. (c) Comparison of flowering time represented by an average number of true rosette leaves at the stage where the flower stem is $1 \mathrm{~cm}$ long. Asterisks in $(\mathbf{a}, \mathbf{b}, \mathbf{c})$ indicate a $\mathrm{p}$-value $<0.05$ or $\mathrm{p}$-value $<0.01$ (double asterisk) (t-test).

Examination of the transcript levels of $F L C, F T, C O$ and SOC1 genes by RT-qPCR showed their transcriptional deregulation. As expected, in many cases reduction in H4K5 acetylation was accompanied by a decreased level of a given transcript (Figure 6c, Figure $7 \mathrm{~b}$ ). In several cases, however, the observed decrease in acetylation did not result in lower transcript levels. In fact, we observed higher relative levels of FT transcript in the Atyaf9a-1 Atyaf $9 b$-2 line, despite a significant decrease of H4K5 acetylation in the promoter region of $F T$ (Figure 6b,c). The lack of clear correlation between H4K5 acetylation and transcriptional activity of tested genes may be at least partially explained by their involvement in a network of functional interactions involving other mechanisms of transcriptional regulation.

\section{Discussion}

In this study, we have investigated the role of a previously uncharacterized protein AtEAF1 as a potential subunit of the plant $\mathrm{NuA} 4$ histone acetylatransferase complex. We found that AtEAF1 and other Arabidopsis homologues of $\mathrm{NuA} 4$ subunits copurify with Arabidopsis homologues of ARP4 and SWC4, common subunits of the yeast NuA4 and SWR1 complexes. Our AtARP4 and AtSWC4 purifications resulted also in detection of peptides belonging to the subunits of the Arabidopsis SWR1 complex including PIE1, which recruits subunits responsible for H2A.Z deposition in Arabidopsis [26,27]. So far, PIE1 has been the only known plant protein with a potential to physically link AtARP4 and AtSWC4 with homologues of other SWR1-C and NuA4 subunits. We argue that the identification of AtEAF1 opens a possibility for a PIE1-independent NuA4 complex formation in plants.

The observed physical association of AtEAF1 with AtAPR4 and AtSWC4 is best explained by the presence of the HSA domain in AtEAF1. HSA domain is a common feature of the platform subunits of SWR1-C, NuA4 and the hybrid complex TIP60-p400. Several published studies suggest that HSA domain may provide the assembly surface for a submodule consisting of ARP4, SWC4, YAF9 and monomeric actin in NuA4 and SWR1 complexes $[5,28,29]$. Indeed, by coimmunoprecipitation we demonstrated a physical interaction between both Arabidopsis YAF9 homologues and a fragment of AtEAF1 containing the HSA domain (Figure 2a). Formation of a protein module by Arabidopsis homologues of ARP4, SWC4 and YAF9 is further supported by the interaction of AtSWC4 with AtYAF9A and AtYAF9B, as revealed by BiFC (Figure 2b,c), and by reciprocal copurification of AtARP4 and AtSWC4, as shown by AP-MS/MS (Table 1). Interestingly, we were not able to detect the expected interaction between YAF9 homologues and the fragment of PIE1 containing the HSA domain in our CoIP assay (Figure 2a). As YAF9 is necessary for H2A.Z deposition in yeast [5,30], it is assumed to be a subunit of the Arabidopsis SWR1-C. Although our CoIP result alone is not sufficient to question this view, it seems to agree with a relatively mild phenotype of the Atyaf9a-1 Atyaf9b-2 mutant (Figure 3) as compared to the pie1-5 or arp6-1 mutant phenotypes [11].

The other conserved sequence feature of AtEAF1 is the SANT domain, located C-terminal of the HSA domain. We found that the combination of HSA and SANT domains is usually represented by no more than two genes per eukaryotic genome (Figure 1c). For example, in both $S$. cerevisiae and human there is just single gene that encodes an HSA-SANT domain protein, i.e. EAF1 and p400, respectively. Importantly, AtEAF1, unlike p400 or PIE1, does not contain an ATPase domain between the HSA and SANT domains. This characteristic leaves EAF1 as the most probable functional analogue of AtEAF1.

Physical interaction of AtEAF1 with AtYAF9A and AtYAF9B is consistent with our observation that plants carrying a T-DNA insertion in one of the AtEAF1 genes are phenotypically similar to Atyaf9a-1 mutants. It has been shown recently that Atyaf9a-1 mutants display reduced levels of $\mathrm{H} 4$ acetylation in $F L C$ gene chromatin [16], which leads to a decrease in FLC transcript levels and, consequently, partial loss of flowering inhibition. Although the double mutant Ateaf1a Ateaf $1 b$ is not currently available, 


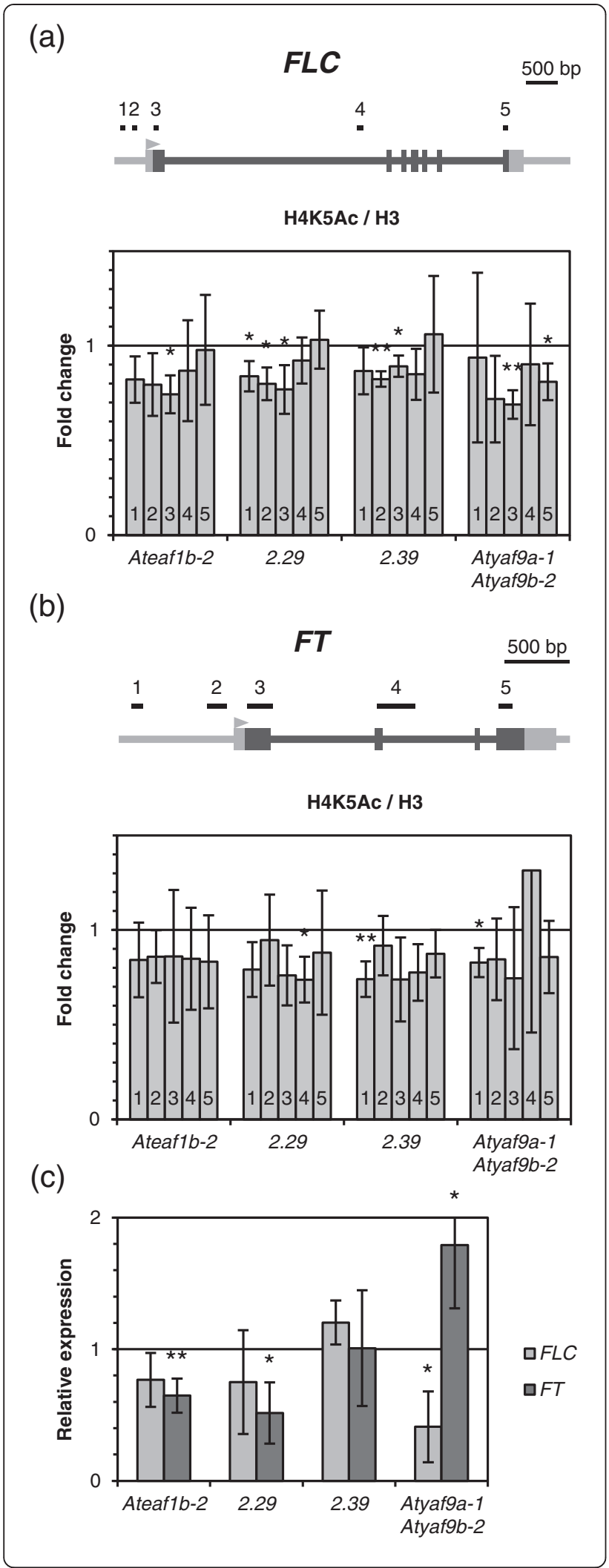

Figure 6 Ateaf1b-2, Atyaf9a-1 Atyaf9b-2 and AtEAF1-amiRNA lines display reduced $\mathrm{H} 4 \mathrm{~K} 5$ acetylation but different activity of $F L C$ and FT. (a, b) Acetylation levels in different parts of the FLC and FT genes normalized to $\mathrm{H} 3$ presented as fold change over WT Col-0. (c) Expression levels of $F L C$ and $F T$ relative to WT Col-0. Asterisks in $(\mathbf{a}, \mathbf{b}, \mathbf{c})$ indicate a $\mathrm{p}$-value $<0.05$ or $\mathrm{p}$-value $<0.01$ (double asterisk) (t-test).

our results show that the single mutant Ateafib-2 is affected in $F L C$ expression and flowering time under LD and SD conditions (Figure 3), phenocopying Atyaf9a-1.

We demonstrated that AtEAF1 and SWC4 interact with both Arabidopsis homologues of YAF9. If the influence of AtYAF9A on FLC expression results from its interaction with AtEAF1, the same could be true for AtYAF9B. Functional redundancy of AtYAF9A and AtYAF9B has been suggested previously on the basis of the phenotype of Atyaf9a-1 Atyaf $9 b-k d$ plants, which differs from the phenotype of either Atyaf9a-1 or Atyaf $9 b-k d$ plants [21]. Our own data show that a double mutant, carrying T-DNA insertions in both YAF9 genes, displays stronger deregulation of flowering time control than either of the single mutants (Figure 3). This result suggests some level of functional redundancy between the two genes in the Arabidopsis NuA4 complex and is in agreement with our finding that both proteins interact with AtEAF1B.

Our analyses of the influence of the histone deacetylase inhibitor TSA revealed an increased resistance to this hyperacetylation-inducing agent in Atyaf $9 a-1$ Atyaf $9 b-2$ and Ateaf1b-2 mutant seedlings. This result supports a role for AtYAF9A, AtYA9B and AtEAF1B in histone acetylation. Under TSA treatment Atyaf $9 a-1$ Atyaf $9 b-2$ accumulated less acetylated H4K5 than WT plants (Additional file 7) which may explain its increased resistance to the drug. No such effect was observed for Ateafb-2 mutant. Several explanations of this result are possible. We chose to follow a hypothesis that AtEAF1 is only required for H4K5 acetylation in specific genomic targets, similar to yeast EAF1 which is mainly required for the NuA4 activity in the promoter regions [31].

As we were not able to determine which genes may be relevant to the negative effect of TSA on plant growth, we used genes encoding main flowering regulators as models to study the role of AtEAF1 in Arabidopsis NuA4. ChIP experiment, in which we employed artificial miRNA knock-down lines for AtEAF1 gene showed that decreased levels of AtEAF1 transcript have similar effect on histone H4K5 acetylation as the disruption of one of the AtEAF1 isoforms in the Ateaf $1 b-2$ mutant (Figures 6 and 7). As expected, we observed a general decrease in H4K5 acetylation levels over most of the tested regions of $F L C$ and $F T$ loci with a slight bias towards their $5^{\prime}$ end (Figure 6a,b). We could also detect significant decrease in acetylation in the promoter of the $C O$ gene and a weaker effect in the SOC1 gene (Figure 7a). 

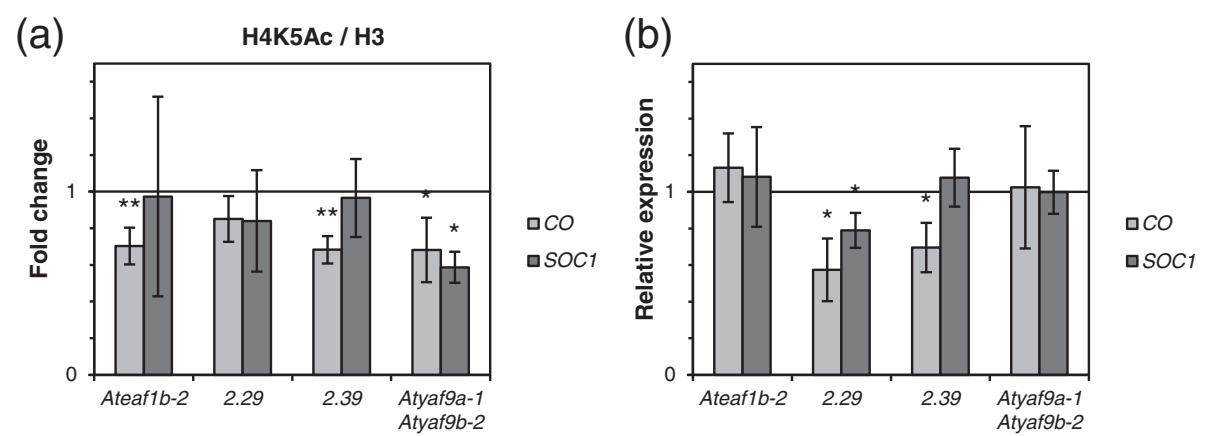

Figure 7 CO is more affected than SOC1 in Ateaf1b-2 and AtEAF1-amiRNA lines. (a) H4K5 acetylation levels in the promoters of CO and SOC1 normalized to H3 presented as fold change over WT Col-0. (b) Expression levels of CO and SOC1 relative to WT Col-0. Asterisks in $(\mathbf{a}, \mathbf{b})$ indicate a $\mathrm{p}$-value $<0.05$ or $\mathrm{p}$-value $<0.01$ (double asterisk) (t-test).

The gene-specific effects which we observed at the level of histone acetylation were not evenly reflected in the transcript levels of the studied genes. This can be at least in part attributed to the functional interactions between FLC, FT, CO, SOC1 and multiple other genes involved in the control of flowering transition. Partial loss of NuA4 activity likely affects many genes with diverse functions. Put to such a stress, the system of positive and negative flowering regulators may seek new balance with outcomes that are difficult to predict. Another possible explanation of these results comes from a recent study on plant homologues of the NuA4 subunit EAF3, i.e. MRG1 and MRG2. In contrast to previous reports showing that Arabidopsis homologues of NuA4 subunits YAF9 and ESA1 contribute to flowering time control mainly by ensuring proper levels of the negative flowering regulator FLC, $\mathrm{Xu}$ et al. demonstrated that transcriptional activity of the positive flowering regulator $F T$ also depends on H4K5 acetylation [17]. According to the report, MRG proteins act as H3K36me3 readers and guide HAM1 and HAM2 acetyltraferases through a direct protein-protein interaction. This does not exclude a role for other $\mathrm{NuA} 4$ subunits as in the case of the yeast $\mathrm{NuA} 4$ complex or human TIP60-p400 which incorporate MRG homologues as stable subunits [12,32]. In fact, MRG1 was among the NuA4 subunits identified as AtSWC4 interaction partners in our AP-MS/MS analyses (Table 1, Additional file 1). Conservation of the AtEAF1 role as a bridge between functional submodules of NuA4 containing YAF9 and EAF3 homologues would explain the ambivalent effects of its partial loss that we observe.

\section{Conclusions}

Our work introduces AtEAF1 as a new subunit of the Arabidopsis NuA4 complex. Characteristic sequence features, interaction with the AtYAF9-AtSWC4-AtARP4 submodule and influence on H4K5 acetylation suggest that AtEAF1 may be a functional analog of the yeast EAF1 protein. Judging from previous studies on human and yeast models, elucidation of the exact role of AtEAF1 subunit in the structural integrity and function of the Arabidopsis NuA4 complex will require significant effort. Our data do not exclude the existence of another large HAT complex in Arabidopsis, organized around PIE1, with additional H2A.Z exchange activity. Addressing this problem may also be required to better understand the regulation of histone $\mathrm{H} 4$ acetylation in plants.

\section{Methods}

\section{Plant material}

The Ateaflb-2 (SALK_067053), Atyaf9a-1 (SALK_106430) and Atyaf9b-2 (SALK_046223) mutants were obtained from the T-DNA mutant collection at Salk Institute. The seeds were provided by Nottingham Arabidopsis Stock Centre (NASC). Positions of T-DNA insertions were confirmed by Sanger sequencing of cloned PCR amplification products (for primer sequences see Additional file 2). The double mutant Atyaf9a-1 Atyaf $9 b-2$ was obtained by crossing the homozygous Atyaf $9 a-1$ and Atyaf $9 b-2$ mutants. WT Columbia-0 ecotype (Col-0) plants were used as a control in all experiments.

\section{Protoplast preparation and transfection}

Protoplasts were prepared from 30-day old plants grown under LD conditions. The Tape-Arabidopsis Sandwich method was used for protoplast preparation [33]. Transfection of protoplasts was carried out in U96 Microwell plates (Thermo Scientific Nunc) according to a published protocol [34].

\section{Coimmunoprecipitation (CoIP)}

For each CoIP, 12 wells in the U96 Microwell plate were used to cotransfect protoplasts with plasmids carrying HSA-Flag and YAF9-nEYFP constructs. Isolation of protoplast proteins and CoIP was performed as previously described [35], except that the immunoprecipitation (IP) lysis buffer was modified by replacing HEPES with $10 \mathrm{mM}$ Tris- $\mathrm{HCl}, \mathrm{pH}$ 7.5. The same buffer was used to wash beads 
after IP. Immunodetection after western blot was done with anti-GFP antibodies (sc-8334, Santa Cruz, www. scbt.com) and anti-Flag antibodies (OctA-Probe Antibody D-8, sc-807, Santa Cruz). All CoIPs were carried out at least twice.

\section{Affinity purification followed by tandem mass spectrometry (AP-MS/MS)}

Expression cassettes containing the coding sequences of the One-STrEP-tag:ATARP4 or One-STrEP-tag:ATSWC4 fusion proteins under the control of the Arabidopsis $U B Q 10$ promoter and NOS terminator were ligated into the binary vector pART27 [36]. Agrobacterium tumefaciens (strain GV3101) mediated transformations were carried out to stably express recombinant proteins in the Arabidopsis suspension-cultured cells (T87 line).

To purify AtARP4 or AtSWC4 complexes, $320 \mathrm{ml}$ ( 24 g of fresh weight) of a 10-day-old T87 culture were vacuum filtered, ground in liquid nitrogen and resuspended in $5 \mathrm{ml}$ of extraction buffer $\mathrm{H}(200 \mathrm{mM} \mathrm{NaCl}, 200 \mathrm{mM}$ Tris- $\mathrm{HCl} \mathrm{pH}$ 8.0, $200 \mathrm{mM} \mathrm{NaF}, 15 \%$ glycerol, $0.3 \mathrm{mM}$ EDTA, 0.5\% TritonX-100, $0.8 \mathrm{mM}$ PMSF, $4 \mathrm{mM}$ DTT, $3.2 \mathrm{mM} \mathrm{NaV}_{2} \mathrm{O}_{3}$,) or $\mathrm{L}(150 \mathrm{mM} \mathrm{NaCl}, 100 \mathrm{mM}$ Tris- $\mathrm{HCl}$ $\mathrm{pH}$ 8.0, $100 \mathrm{mM}$ NaF, 10\% glycerol, $2.5 \mathrm{mM}$ EDTA, 2.5 mM EGTA, 0.4\% Triton X-100, $0.8 \mu \mathrm{M}$ PMSF, $4 \mathrm{mM}$ DTT, $3.2 \mathrm{mM} \mathrm{NaV}_{2} \mathrm{O}_{3}$ ) with one tablet of cOmplete EDTA-free Protease Inhibitor Cocktail (Roche, www.roche. com). After centrifugation $\left(15000 \mathrm{~g}, 15 \mathrm{~min}, 4^{\circ} \mathrm{C}\right.$ and then $180000 \mathrm{~g}, 90 \mathrm{~min}, 4^{\circ} \mathrm{C}$ ) supernatant was loaded onto a column containing $600 \mu \mathrm{l}$ of Strep-Tactin Superflow High Capacity resin (IBA). After seven washing steps (100 mM Tris- $\mathrm{HCl} \mathrm{pH} 8.0,150 \mathrm{mM} \mathrm{NaCl}$ ), bound proteins were eluted with $1.8 \mathrm{ml}$ of $2.5 \mathrm{mM}$ desthiobiotin (IBA) and finally concentrated and desalted using Amicon Ultra $10 \mathrm{~K}$ (Millipore, www.millipore.com).

Trypsin-digested peptides were analyzed using Orbitrap Velos (Thermo Scientific, www.thermo.com) coupled with nanoAcquity UPLC (Waters, www.waters.com) according to standard protocols at the Mass Spectrometry Laboratory, Institute of Biochemistry and Biophysics, Polish Academy of Sciences, Warsaw. To identify proteins, Mascot software (Matrix Science, www.matrixscience. com) was employed to search mass spectra against the TAIR10 database using the following parameters: no missed cleavages allowed, $20 \mathrm{ppm}$ for peptide mass tolerance and $0.6 \mathrm{Da}$ for fragment ion mass tolerance, fixed modification - cysteine carbamidomethylation, variable modification - methionine oxidation. Results were filtered using significance threshold $\mathrm{p}$-value $<0.05$ and expect cut-off 0.05 (http://www.matrixscience.com/ help/scoring_help.html).

A. thaliana T87 suspension-cultured cells were grown at $22^{\circ} \mathrm{C}$ under continuous light $\left(50 \mu \mathrm{mol} \mathrm{m} \mathrm{m}^{-2} \mathrm{~s}^{-1}\right)$ and shaking at $120 \mathrm{rpm}$ in Gamborg's B5 medium [37] with
1.5\% sucrose and $0.1 \mu \mathrm{g} / 1$ 2,4-dichlorophenoxyacetic acid (Sigma-Aldrich, www.sigmaaldrich.com).

\section{Bimolecular fluorescence complementation (BiFC)}

For BiFC, single wells in the U96 Microwell plate were used to transfect protoplasts with plasmids encoding proteins of interest fused to the N-terminus of nEYFP and cEYFP. Empty vector $(3 \mu \mathrm{g})$ encoding Enhanced Cyan Fluorescent Protein (ECFP) was added to each well as a transfection control. After transfection, protoplasts resuspended in $200 \mu \mathrm{l}$ of W5 solution with $5 \mathrm{mM}$ glucose [33] were transferred to black 96-well black glass-bottom plates (SensoPlate, Greiner Bio-One, www.greinerbioone.com), sealed with transparent sealing tape (Thermo Scientific Nunc, cat. no. 236366), and returned to the growth chamber for overnight incubation. After incubation, the sealing tape was removed and the plate was screened with a Nikon A1Rsi confocal system (www.nikon.com). See Additional file 8 for original files.

\section{Gene expression analyses}

RNA was extracted using TRI Reagent (Sigma-Aldrich). For the analysis of FLC expression, RNA was extracted from 10- and 16-day old seedlings, grown in plates under LD (16 hours of light) or SD (8 hours of light) conditions, respectively. For the analysis of relative expression levels of AtEAF1A and AtEAF1B, RNA was extracted from mature rosette leaves of Col-0 WT plants grown under LD conditions. The gene expression data presented in Figures 6 and 7 was obtained from plants grown and harvested in the same way as plants grown for ChIP analyses. Reverse transcription was done with the RevertAid kit (Thermo Scientific). Relative expression levels of FLC were measured by quantitative PCR. UBQ10 (Figures 3 and 5) and $U B C 21$ (Figures 6 and 7) gene transcripts were used as a reference. The $2^{-\Delta \Delta C t}$ method [38] was used to quantify the relative transcript levels in all experiments. Sequences of all primers can be found in Additional file 2.

\section{Preparation of genetic constructs}

Coding sequences of AtYAF9B, AtEAF1, PIE1 and INO80 were cloned from cDNA obtained from WT Col-0 plants. The remaining coding sequences were subcloned from pUNI51 constructs from the FL-cDNA collection at the Salk Institute, provided by Arabidopsis Biological Resource Center (ABRC). All transient expression vectors used in this study are based on $\mathrm{PSAT}$ vector series [39]. The Flag vectors were constructed by replacing the nEYFP sequence with Flag sequence. All vectors were modified by inserting two Sfil recognition sites into the multiple cloning site to facilitate transfer of coding sequences between vectors. Sequences of all primers can be found in Additional file 2. 


\section{Sequence analyses}

Conserved protein domains were found using InterProScan [40]. Domain relatives were identified using CDART [41] and by sequence similarity search using BLAST (blast.ncbi. nlm.nih.gov). All multiple sequence alignments were performed using the T-Coffee program [42] accessed through EMBL-EBI web services (www.ebi.ac.uk). For visualization of sequence alignment data, Geneious version 6.0 was used (Biomatters, www.geneious.com).

\section{Trichostatin A treatment}

Plants were grown in plates containing MS medium supplemented with $1 \%$ sucrose with $\mathrm{pH}$ stabilized by MES at 5.7. DMSO solution of Trichostatin A (Sigma-Aldrich) was added to the medium before pouring the plates to the final concentration of $12.5 \mu \mathrm{M}$. Equal amount of DMSO was added as a mock. Seeds were surface-sterilized before sawing and kept in $4^{\circ} \mathrm{C}$ for four days. After stratification, plates were transferred to a growth chamber with long day conditions (16 h of light). The measurements were done on 12-day old seedlings.

\section{Artificial micro RNA lines}

Artificial micro RNA transgene was designed with the WMD3 tool (wmd3.weigelworld.org) and constructed according to the instructions available at the same website. Briefly, the target-specific sites of the Arabidopsis miRNA precursor MIR319a cloned into pRS300 vector were mutated through overlap extension PCR to target a sequence identical in both AtEAF1 genes. pRS300 was a gift from Detlef Weigel (Addgene plasmid \# 22846). After confirmation of the mutations by DNA sequencing, the precursor was placed downstream of the UBQ10 promoter in a binary vector using standard molecular biology procedures. The plasmid was then transferred into Agrobacterium tumefaciens strain GV3101. A. thaliana Col-0 plants were transformed using floral deep method [43]. Transgenic plants were selected on soil by spraying with diluted BASTA (Bayer). Presence of the amiRNA construct was confirmed by PCR. Progeny of the BASTA resistant plants of the $\mathrm{T} 1$ and $\mathrm{T} 2$ plants was used in subsequent experiments. Sequences of all primers can be found in Additional file 2 .

\section{Chromatin immunoprecipitation (ChIP)}

About $350 \mathrm{mg}$ of 10-day-old seedlings grown in LD conditions were harvested at the end of the light photoperiod, ground in liquid nitrogen and then fixed for $15 \mathrm{~min}$ at room temperature in extraction buffer containing 1\% formaldehyde (60 mM Hepes $\mathrm{pH}$ 8.0, $1 \mathrm{M}$ sucrose, $5 \mathrm{mM} \mathrm{KCl}, 5 \mathrm{mM} \mathrm{MgCL} 2,5 \mathrm{mM}$ EDTA, $0.6 \%$ Triton X-100, 1\% formaldehyde, 1 mM PMSF, 1\% Protease Inhibitor Cocktail, Sigma). The reaction was quenched by adding glycine to a final concentration of $100 \mathrm{mM}$. The homogenate was filtered through Miracloth, centrifuged and the pellet was washed with buffer containing $0.25 \mathrm{M}$ sucrose, $10 \mathrm{mM}$ Tris- $\mathrm{HCl} \mathrm{pH}$ 8.0, $10 \mathrm{mM} \mathrm{MgCl} 2,1 \%$ Triton X-100, 1 mM EDTA, 5 mM beta-mercaptoethanol, $1 \mathrm{mM}$ PMSF and Protease Inhibitor Cocktail. Then, the pellet was resuspended in the sonication buffer $(50 \mathrm{mM}$ Tris-HCL pH 8.0, 10 mM EDTA, 0.5\% SDS, 1 mM PMSF and Protease Inhibitor Cocktail) and sonicated using Bioruptor (Diagenode) for 25 cycles of $30 \mathrm{sec}$ on and $30 \mathrm{sec}$ off. Sonicated chromatin was diluted 10-fold and incubated with antibodies (anti-H3, Abcam, ab1791; anti$\mathrm{H} 4 \mathrm{~K} 5 \mathrm{ac}$, Millipore 07-327) overnight at $4^{\circ} \mathrm{C}$ with gentle agitation. After pre-clearing, magnetic protein A-beads (Dynabeads protein A, Life Sciences) were incubated with the antibodies-chromatin mix for 1 hours. The slurry was then washed and DNA was extracted with 10\% Chelex (Biorad) as described previously [44].

All ChIP experiments were carried out with three independent biological replicates and quantified by qPCR (Maxima SybrGreen/ROX, Thermo Scientific). Primer sequences used for the ChIP-qPCR are listed in Additional file 2. The ChIP data are presented as the ratio of percent of input for $\mathrm{H} 4 \mathrm{~K} 5 \mathrm{ac}$ to percent of input for total $\mathrm{H} 3$ normalized to WT levels. Error bars correspond to SD of the mean of three biological replicates. Statistical evaluations were performed using a Student's t-test.

\section{Additional files}

\section{Additional file 1: AP-MS/MS data.}

Additional file 2: Oligonucleotides used in this study. Additional file 3: DNA and protein sequences, alignments. Additional file 4: Gene structures of AtYAF9A, AtYAF9B and AtEAF1B with positions of T-DNA insertions in the mutant lines marked. Details of the amiRNA design and phenotypes of the silenced lines. Relative expression levels of the AtEAFIA and AtEAF1B genes.

Additional file 5: Uncropped scans of the Western blot films.

Additional file 6: BiFC screening for protein-protein interactions between AtARP4, AtSWC4, AtYAF9A and AtYAF9B.

Additional file 7: Western Blot analyses of bulk H4K5 acetylation levels in mutants and WT plants treated or untreated with TSA.

Additional file 8: Original confocal images (.nd2 format requires free viewer available at www.nikoninstruments.com/Products/ Software/NIS-Elements-Viewer).

\section{Competing interests}

The authors declare that they have no competing interests.

\section{Authors' contributions}

TB drafted the manuscript, developed all main concepts of the work, designed or participated in designing and data analysis of all experiments, performed all sequence analyses, prepared all genetic constructs except those used in the AP-MS/MS, performed BiFC and TSA assays, participated in CoIP, RT-qPCR and phenotypic analyses. LG designed and performed the AP-MS/MS experiments and provided crucial ideas for the modification of the PSAT vectors. AB participated in designing the CoIP experiment and carried out all work on the proteins overexpressed in protoplasts. MA obtained the double mutant Atyaf9a-1 Atyaf9b-2, performed major part of the phenotypic analyses and participated in the RT-qPCR analyses. 
WS participated in the phenotypic analyses, designed and carried out the ChIP experiments. AL participated in the data analysis and discussions. PAZ and JS provided the conceptual background and supported TB in all experimental designs, interpretation of the results and preparation of the manuscript. All authors read and approved the final manuscript.

\section{Acknowledgements}

This work was supported by the Polish National Science Center NCN grants (NCN UMO-2011/01/B/NZ2/01691 to J.S. and N N303 313437 to P.A.Z.) and KNOW RNA Research Centre in Poznan, 01/KNOW2/2014. The PhD fellowship of T.B. is part of the International PhD Programme 'From genome to phenotype: A multidisciplinary approach to functional genomics' (MPD/2010/3) of the Foundation for Polish Science (FNP), cofinanced from European Union, Regional Development Fund (Innovative Economy Operational Programme 2007-2013). T.B. would like to thank Jerzy Paszkowski and Marco Catoni for useful suggestions and Daniel Kierzkowski for the introduction to the protoplast expression system and BiFC.

\section{Author details}

${ }^{1}$ Department of Biotechnology, Institute of Molecular Biology and Biotechnology, Adam Mickiewicz University, Umultowska 89, 61-614 Poznań, Poland. ${ }^{2}$ Department of Molecular Virology, Institute of Experimental Biology, Adam Mickiewicz University, Umultowska 89, 61-614 Poznań, Poland. ${ }^{3}$ Department of Plant Sciences, University of Cambridge, Downing Street, CB2 3EA, Cambridge, UK.

\section{Received: 12 April 2014 Accepted: 13 February 2015}

\section{Published online: 05 March 2015}

\section{References}

1. Cairns BR, Schlichter A, Erdjument-Bromage H, Tempst P, Kornberg RD, Winston F. Two functionally distinct forms of the RSC nucleosome-remodeling complex, containing essential at hook, BAH, and bromodomains. Mol Cell. 1999:4:715-23.

2. Chandy M, Gutiérrez JL, Prochasson P, Workman JL. SWI/SNF Displaces SAGA-Acetylated Nucleosomes. Eukaryot Cell. 2006:5:1738-47.

3. Papamichos-Chronakis M, Watanabe S, Rando OJ, Peterson CL. Global Regulation of H2A.Z Localization by the INO80 Chromatin-Remodeling Enzyme Is Essential for Genome Integrity. Cell. 2011;144:200-13.

4. Altaf M, Auger A, Monnet-Saksouk J, Brodeur J, Piquet S, Cramet M, et al. NuA4-dependent Acetylation of Nucleosomal Histones $\mathrm{H} 4$ and H2A Directly Stimulates Incorporation of H2A.Z by the SWR1 Complex. J Biol Chem. 2010;285:15966-77.

5. Auger A, Galarneau L, Altaf M, Nourani A, Doyon Y, Utley RT, et al. Eaf1 is the platform for NuA4 molecular assembly that evolutionarily links chromatin acetylation to ATP-dependent exchange of histone H2A variants. Mol Cell Biol. 2008:28:2257-70.

6. Mizuguchi G, Shen X, Landry J, Wu W-H, Sen S, Wu C. ATP-driven exchange of histone H2AZ variant catalyzed by SWR1 chromatin remodeling complex. Science. 2004;303:343-8.

7. Wu W-H, Wu C-H, Ladurner A, Mizuguchi G, Wei D, Xiao H, et al. N Terminus of Swr1 binds to Histone $\mathrm{H} 2 \mathrm{AZ}$ and provides a platform for subunit assembly in the chromatin remodeling complex. J Biol Chem. 2009;284:6200-7.

8. Gévry N, Chan HM, Laflamme L, Livingston DM, Gaudreau L. p2 transcription is regulated by differential localization of histone H2A.Z. Genes Dev. 2007:21:1869-81.

9. Park JH, Sun X-J, Roeder RG. The SANT Domain of p400 ATPase Represses Acetyltransferase Activity and Coactivator Function of TIP60 in Basal p21 Gene Expression. Mol Cell Biol. 2010;30:2750-61.

10. Noh Y-S, Amasino RM. PIE1, an ISWI Family Gene, Is Required for FLC Activation and Floral Repression in Arabidopsis. Plant Cell Online. 2003;15:1671-82.

11. Deal RB, Topp CN, McKinney EC, Meagher RB. Repression of flowering in Arabidopsis requires activation of FLOWERING LOCUS C expression by the histone variant H2A.Z. Plant Cell. 2007;19:74-83.

12. Doyon Y, Selleck W, Lane WS, Tan S, Côté J. Structural and functional conservation of the NuA4 histone acetyltransferase complex from yeast to humans. Mol Cell Biol. 2004:24:1884-96.

13. Earley KW, Shook MS, Brower-Toland B, Hicks L, Pikaard CS. In vitro specificities of Arabidopsis co-activator histone acetyltransferases: implications for histone hyperacetylation in gene activation. Plant J. 2007;52:615-26.
14. Latrasse D, Benhamed M, Henry Y, Domenichini S, Kim W, Zhou D-X, et al. The MYST histone acetyltransferases are essential for gametophyte development in Arabidopsis. BMC Plant Biol. 2008;8:121.

15. Xiao J, Zhang H, Xing L, Xu S, Liu H, Chong $K$, et al. Requirement of histone acetyltransferases HAM1 and HAM2 for epigenetic modification of FLC in regulating flowering in Arabidopsis. J Plant Physiol. 2013;170:444-51.

16. Zacharaki V, Benhamed M, Poulios S, Latrasse D, Papoutsoglou P, Delarue $\mathrm{M}$, et al. The Arabidopsis ortholog of the YEATS domain containing protein YAF9a regulates flowering by controlling $\mathrm{H} 4$ acetylation levels at the FLC locus. Plant Sci. 2012;196:44-52.

17. Xu Y, Gan E-S, Zhou J, Wee W-Y, Zhang X, Ito T. Arabidopsis MRG domain proteins bridge two histone modifications to elevate expression of flowering genes. Nucleic Acids Res. 2014;42:10960-74.

18. Shen X, Mizuguchi G, Hamiche A, Wu C. A chromatin remodelling complex involved in transcription and DNA processing. Nature. 2000;406:541-4.

19. Jin J, Cai Y, Yao T, Gottschalk AJ, Florens L, Swanson SK, et al. A mammalian chromatin remodeling complex with similarities to the yeast INO80 complex. J Biol Chem. 2005;280:41207-12.

20. Vercruyssen L, Verkest A, Gonzalez N, Heyndrickx KS, Eeckhout D, Han S-K, et al. ANGUSTIFOLIA3 binds to SWI/SNF chromatin remodeling complexes to regulate transcription during Arabidopsis leaf development. Plant Cell. 2014:26:210-29.

21. Choi K, Kim J, Hwang H-J, Kim S, Park C, Kim SY, et al. The FRIGIDA Complex Activates Transcription of FLC, a Strong Flowering Repressor in Arabidopsis, by Recruiting Chromatin Modification Factors. Plant Cell Online. 2011;23:289-303.

22. Bittner CB, Zeisig DT, Zeisig BB, Slany RK. Direct Physical and Functional Interaction of the NuA4 Complex Components Yaf9p and Swc4p. Eukaryot Cell. 2004:3:976-83.

23. Yang F, Zhang L, Li J, Huang J, Wen R, Ma L, et al. Trichostatin A and 5-azacytidine both cause an increase in global histone $\mathrm{H} 4$ acetylation and a decrease in global DNA and H3K9 methylation during mitosis in maize. BMC Plant Biol. 2010;10:178.

24. Tanaka M, Kikuchi A, Kamada H. The Arabidopsis Histone Deacetylases HDA6 and HDA19 Contribute to the Repression of Embryonic Properties after Germination. Plant Physiol. 2008;146:149-61.

25. Schwab R, Ossowski S, Riester M, Warthmann N, Weigel D. Highly Specific Gene Silencing by Artificial MicroRNAs in Arabidopsis. Plant Cell Online. 2006;18:1121-33.

26. Choi K, Park C, Lee J, Oh M, Noh B, Lee I. Arabidopsis homologues of components of the SWR1 complex regulate flowering and plant. Development. 2007;134:1931-41.

27. March-Díaz R, García-Domínguez M, Florencio FJ, Reyes JC. SEF, a New Protein Required for Flowering Repression in Arabidopsis, Interacts with PIE1 and ARP6. Plant Physiol. 2007;143:893-901.

28. Szerlong $H$, Hinata $K$, Viswanathan $R$, Erdjument-Bromage $H$, Tempst $P$, Cairns BR. The HSA domain binds nuclear actin-related proteins to regulate chromatin-remodeling ATPases. Nat Struct Mol Biol. 2008;15:469-76.

29. Nguyen VQ, Ranjan A, Stengel F, Wei D, Aebersold R, Wu C, et al. Molecular architecture of the ATP-dependent chromatin-remodeling complex SWR1. Cell. 2013;154:1220-31.

30. Zhang H, Richardson DO, Roberts DN, Utley R, Erdjument-Bromage $H$, Tempst P, et al. The Yaf9 component of the SWR1 and NuA4 complexes is required for proper gene expression, histone $\mathrm{H} 4$ acetylation, and $\mathrm{Htz} 1$ replacement near telomeres. Mol Cell Biol. 2004;24:9424-36.

31. Ginsburg DS, Govind CK, Hinnebusch AG. NuA4 lysine acetyltransferase esa1 is targeted to coding regions and stimulates transcription elongation with Gcn5. Mol Cell Biol. 2009;29:6473-87.

32. Eisen A, Utley RT, Nourani A, Allard S, Schmidt P, Lane WS, et al. The yeast NuA4 and drosophila MSL complexes contain homologous subunits important for transcription regulation. J Biol Chem. 2001;276:3484-91.

33. Wu F-H, Shen S-C, Lee L-Y, Lee S-H, Chan M-T, Lin C-S. Tape-Arabidopsis sandwich - a simpler Arabidopsis protoplast isolation method. Plant Methods. 2009;5:16.

34. Fujikawa Y, Kato N. TECHNICAL ADVANCE: split luciferase complementation assay to study protein-protein interactions in Arabidopsis protoplasts. Plant J. 2007:52:185-95.

35. Li J-F, Bush J, Xiong Y, Li L, McCormack M. Large-Scale Protein-Protein Interaction Analysis in Arabidopsis Mesophyll Protoplasts by Split Firefly Luciferase Complementation. PLOS ONE. 2011;6

36. Gleave AP. A versatile binary vector system with a T-DNA organisational structure conducive to efficient integration of cloned DNA into the plant genome. Plant Mol Biol. 1992;20:1203-7. 
37. Yamada H, Koizumi N, Nakamichi N, Kiba T, Yamashino T, Mizuno T. Rapid response of Arabidopsis T87 cultured cells to cytokinin through his-to-Asp phosphorelay signal transduction. Biosci Biotechnol Biochem. 2004;68:1966-76.

38. Livak KJ, Schmittgen TD. Analysis of relative gene expression data using real-time quantitative $P C R$ and the $2-\Delta \Delta C T$ method. Methods. 2001;25:402-8.

39. Tzfira T, Tian G-W, Lacroix B, Vyas S, Li J, Leitner-Dagan Y, et al. pSAT vectors: a modular series of plasmids for autofluorescent protein tagging and expression of multiple genes in plants. Plant Mol Biol. 2005;57:503-16.

40. Quevillon E, Silventoinen V, Pillai S, Harte N, Mulder N, Apweiler R, et al. InterProScan: protein domains identifier. Nucleic Acids Res. 2005;33 suppl 2:W116-20.

41. Geer LY, Domrachev M, Lipman DJ, Bryant SH. CDART: protein homology by domain architecture. Genome Res. 2002;12:1619-23.

42. Notredame C, Higgins DG, Heringa J. T-coffee: a novel method for fast and accurate multiple sequence alignment. J Mol Biol. 2000;302:205-17.

43. Clough SJ, Bent AF. Floral dip: a simplified method forAgrobacterium-mediated transformation ofArabidopsis thaliana. Plant J. 1998;16:735-43.

44. Wierzbicki AT, Haag JR, Pikaard CS. Noncoding transcription by RNA Polymerase Pol IVb/Pol V mediates transcriptional silencing of overlapping and adjacent genes. Cell. 2008;135:635-48.

\section{Submit your next manuscript to BioMed Central and take full advantage of:}

- Convenient online submission

- Thorough peer review

- No space constraints or color figure charges

- Immediate publication on acceptance

- Inclusion in PubMed, CAS, Scopus and Google Scholar

- Research which is freely available for redistribution 